\title{
DISCRETE O(1,4) TRANSFORMATIONS AND NEW SCALAR QUANTUM MODES ON THE DE SITTER SPACETIME
}

\author{
Ion I. Cotăescu
}

West University of Timişoara, V. Parvan Ave. 4, RO-300223 Timişoara, Romania

\section{Article Info \\ Received: 28 December 2011 \\ Accepted: 19 January 2012}

Keywords: de Sitter; isometries; discrete isometries; scalar quantum modes Pacs: 04.20.Cv, 04.62.+v, 11.30.-j

\begin{abstract}
It is shown that the isometry group of the de Sitter spacetime includes two different three-dimensional Abelian subgroups which transform between themselves through a discrete isometry corresponding to the time reversal in the five-dimensional Minkowski spacetime embedding the de Sitter one. The eigenfunctions of the generators of these Abelian subgroups form two different sets of quantum modes correlated by the mentioned isometry.
\end{abstract}

The quantum theory of fields on the de Sitter spacetime has the advantage of the $S O(1,4)$ symmetry [1] which provides one with the conserved $s o(1,4)$ generators commuting with the operators of the field equations [2]. These differential operators represent the principal quantum observables which enable one to define quantum modes as common eigenfunctions of several sets of commuting operators including that of the field equation. However, the physical meaning of these generators is not fully understood even though in the flat limit one recovers the Poincaré symmetry [2]. An open problem is related to the presence in $S O(1,4)$ of two Abelian subgroups $T(3)$ among them only one can be interpreted as the subgroup of space translations in the (co)moving charts [3]. The role of the second $T(3)$ subgroup remains obscure as long as its generators are less studied so far. For this reason we would like to analyze here the relation between these two $T(3)$ subgroups investigating the properties of the mode functions defined as eigenfunctions of their generators. Our principal result points out that these subgroups transform between themselves through a discrete $O(1,4)$ de Sitter isometry corresponding to the time reversal in the five-dimensional Minkowski spacetime embedding the de Sitter one. 
Let us start with the de Sitter spacetime $(M, g)$ defined as the hyperboloid of radius $1 / \omega^{1}$ in the five-dimensional flat spacetime $\left(M^{5}, \eta^{5}\right)$ of coordinates $z^{A}$ (labeled by the indices $A, B, \ldots=0,1,2,3,4)$ and metric $\eta 5=\operatorname{diag}(1,-1,-1,-1,-1)[1]$. A local chart of coordinates $x^{\mu}$ $(\mu, v, \ldots=0,1,2,3)$ can be introduced on $(M, g)$ giving the set of functions $z^{A}(x)$ which solve the hyperboloid equation, $\eta_{A B}^{5} z^{A}(x) z^{B}(x)=-\omega^{-2}$. In this manner $(M, g)$ is defined as a homogeneous space of the pseudo-orthogonal group $O(1,4)$. The proper $O(1,4)$ transformations form the subgroup $S O(1,4)$ while the improper ones have to be written using discrete transformations on $M^{5}$.

The group $S O(1,4)$ is in the same time the gauge group of the metric $\eta^{5}$ and the isometry group, $I(M)$, of the de Sitter spacetime. Its universal covering group, $\mathrm{S}(\mathrm{M})=$ $\operatorname{Spin}(\eta 5)=\operatorname{Sp}(2,2)$, is the group of external symmetry as defined in Ref. [4]. This has the Lie algebra $s(M)=s p(2,2) \sim s o(1,4)$ for which we use the covariant real parameters $\xi^{A B}=-\xi^{B A}$. In this parametrization, any $\Lambda(\xi) \in S O(1,4)$ produces the linear transformation $z^{A}(x) \rightarrow z^{A}\left(x^{\prime}\right)=\Lambda(\xi) A \cdot, \cdot B_{z}^{B}(x) \quad$ giving rise to the isometry $x \rightarrow x^{\prime}=\phi_{\xi}(x)=x+k_{A B}(x) \xi^{A B}+\ldots$ which can be expanded in terms of Killing vectors (of components $k \mu, A B$ ) associated to the parameters $\xi^{A B}$. These Killing vectors allow one to write down the basis-generators of the covariant representations according to which the matter fields transform under isometries $[5,4,2]$. In the simplest case of the scalar fields $\psi$, which transform according to the natural representation $\psi \rightarrow \psi^{\prime}=\psi \circ \phi_{\xi}^{-1}$, these generators are the orbital operators $L_{A B}=-i k_{A B}^{\mu} \partial_{\mu}[6,7]$.

We assume now that $(M, g)$ is equipped with the local chart $\{t, \vec{x}\}$ of conformal time $t$ and Cartesian space coordinates defined by the functions

$$
\begin{array}{r}
z^{0}(x)=-\frac{1}{2 \omega^{2} t}\left[1-\omega^{2}\left(t^{2}-\vec{x}^{2}\right)\right] \\
z^{4}(x)=-\frac{1}{2 \omega^{2} t}\left[1+\omega^{2}\left(t^{2}-\vec{x}^{2}\right)\right] \\
z^{i}(x)=-\frac{x^{i}}{\omega t^{\prime}} \quad i, j, \ldots=1,2,3,
\end{array}
$$

giving rise to the conformal-flat line element

\footnotetext{
${ }^{1}$ We denote by $\omega$ the Hubble de Sitter constant since $H$ is reserved for the Hamiltonian operator
} 


$$
d s^{2}=\eta_{A B}^{5} d z^{A} d z^{B}=\frac{1}{\omega^{2} t^{2}}\left(d t^{2}-d \vec{x} \cdot d \vec{x}\right)
$$

This chart covers the expanding part of $M$ for $t \in(-\infty, 0)$ and $\vec{x} \in R^{3}$ while the collapsing part is covered by a similar chart with $t>0$ [3].

In these charts it is convenient to use the basis $\left\{H, P_{i}, Q_{i}, L_{i}\right\}$ of the natural representation of the $s o(1,4)$ algebra which is formed by the energy operator $H \equiv \omega L_{(04)}=-i \omega\left(t \partial_{t}+x^{i} \partial_{i}\right)$, the momentum operator $\vec{P}$ and its dual, $\vec{Q}$, whose components are defined as [2]

$$
\begin{gathered}
P_{i} \equiv \omega\left(L_{(i 4)}-L_{(i 0)}\right)=i \partial_{i}, \\
Q_{i} \equiv \omega\left(L_{(i 4)}+L_{(i 0)}\right)=2 i x^{i} \omega^{2}\left(t \partial_{t}+x^{i} \partial_{j}\right)+i \omega^{2}\left(t^{2}-\vec{x}^{2}\right) \partial_{i},
\end{gathered}
$$

and the angular momentum $L_{i}=\frac{1}{2} \varepsilon_{i j k} L_{(j k)}=-i \varepsilon_{i j k} x^{i} \partial_{k}$. These generators satisfy the commutation relations [2]

$$
\begin{aligned}
& {\left[H, P_{i}\right]=i \omega P_{i}, \quad\left[H, Q_{i}\right]=-i \omega Q_{i},} \\
& {\left[L_{i}, P_{i}\right]=i \varepsilon_{i j k} P_{k}, \quad\left[L_{i}, Q_{j}\right]=i \varepsilon_{i j k} Q_{k}} \\
& {\left[P_{i}, P_{j}\right]=0, \quad\left[Q_{i}, Q_{j}\right]=0,} \\
& {\left[Q_{i}, P_{j}\right]=2 i \omega \delta_{i j} H+2 i \omega^{2} \varepsilon_{i j k} L_{k}}
\end{aligned}
$$

The first Casimir operator of this algebra coincides to the Klein-Gordon operator, $C_{1}=H^{2}+3 i \omega H-\vec{Q} \cdot \vec{P}-\omega^{2} L^{2}=\varepsilon_{K G}$, while the second one, $C_{2}=0$, vanishes since the there is no spin [2]. For this reason the usual scalar quantum modes, called here $P$-modes, are determined as common eigenfunctions of the set of commuting operators $\left\{\varepsilon_{K G}, P_{i}\right\}$ [7].

On the other hand, it is remarkable that the operators $Q_{i}$ form the subalgebra of an Abelian subgroup $T(3){ }_{Q}$ isomorphic to the subgroup $T(3)_{P}$ of the physical translations in the chart $\{t, \vec{x}\}$ generated by $P_{i}$. This suggests us that new quantum modes, called $Q$-modes, could be defined as common eigenfunctions of the set $\left\{\varepsilon_{K G}, Q_{i}\right\}$. These may be written down by using a coordinate transformation able to change the Abelian generators among themselves $\left(P_{i} \leftrightarrow Q_{i}\right)$ without to affect the other generators. This transformation can not be an 
$S O(1,4)$ isometry since the $s o(1,4)$ generators $L_{A B}$ transform under isometries as skewsymmetric tensors. This means that we are left only with the discrete transformations of the group $O(1,4)$ which need our attention in what follows.

The simplest discrete transformations on $M^{5}$, denoted by $\Pi_{[A]}$, are those which change the sign of a single coordinate, $z^{A} \rightarrow-z^{A}$. These transformations give rise to the discrete isometries $x \rightarrow x^{\prime}=\pi_{[A]}(x)$ defined as $\Pi_{[A]} z(x)=z\left[\pi_{[A]}(x)\right]$. Obviously, these isometries satisfy $\pi_{[A]} \circ \pi_{[A]}=i d$ where $i d$ denote the identity function. A rapid inspection indicates that the interesting non-trivial isometries are produced by $\Pi_{[0]}$ as

$$
t^{\prime}=\pi_{[0]}^{0}(x)=-\frac{t}{\omega^{2}\left(t^{2}-\vec{x}^{2}\right)}, \quad x^{\prime i}=\pi_{[0]}^{i}(x)=-\frac{x^{i}}{\omega^{2}\left(t^{2}-\vec{x}^{2}\right)} .
$$

and by $\Pi_{[4]}$ which gives

$$
t^{\prime}=\pi_{[4]}^{0}(x)=-\frac{t}{\omega^{2}\left(t^{2}-\vec{x}^{2}\right)}, \quad x^{\prime i}=\pi_{[4]}^{i}(x)=-\frac{x^{i}}{\omega^{2}\left(t^{2}-\vec{x}^{2}\right)} .
$$

The other isometries, $\pi_{[i]}$, are simple mirror transformations of the space coordinates $x^{i}$ such that the (space) parity reads $\pi_{[x]}=\pi_{[1]} \circ \pi_{[2]} \circ \pi_{[3]}$. Another remarkable discrete isometry is $\pi_{[x]}=\pi_{[0]}{ }^{\circ} \pi_{[4]}$ which changes the signs of all the coordinates $x^{\mu}$. It is worth noting that the physical measurements can be performed only inside the light-cone where $|t|>|\vec{x}|$. This indicates that the isometry (11) does not change the signs of the time and space coordinates and, consequently, the charts $\{t, \vec{x}\}$ and $\left\{t^{\prime}, \vec{x}^{\prime}\right\}$ cover the same portion of $M$. On the contrary, the isometry (12) changes the sign of the conformal time moving the transformed chart to the opposite portion of this manifold.

Now our problem can be solved observing that the transformation $\Pi_{[0]}$ changes only the signs of the generators $L_{(0 i)}$ and $L_{(04)}$ so that, in the chart $\left\{t^{\prime}, \vec{x}^{\prime}\right\}$ defined by equations (11), we meet the new basis-generators

$$
P_{i}^{\prime}=Q_{i}, Q_{i}^{\prime}=P_{i}, H^{\prime}=-H, L_{i}^{\prime}=L_{i}
$$

but the same Casimir operator $\varepsilon_{K G}^{\prime}=\varepsilon_{K G}$ since $3 i \omega H^{\prime}-\vec{Q}^{\prime} \cdot \vec{P}^{\prime}=3 i \omega H-\vec{Q} \cdot \vec{P}$ as it results from equations (10) and (13). Therefore, the $P$-mode functions $f_{\vec{p}}(x)$ of the chart $\{t, \vec{x}\}$, which satisfy the Klein-Gordon equation $\varepsilon_{K G} f_{\vec{p}}(x)=m^{2} f_{\vec{p}}(x)$ and the eigenvalue 
problems $P_{i} f_{\vec{p}}(x)=p_{i} f_{\vec{p}}(x)$, become $Q$-mode functions in the chart $\left\{t^{\prime}, \vec{x}^{\prime}\right\}$ where these obey $\varepsilon_{K G}^{\prime} f_{\vec{p}}\left(x^{\prime}\right)=m^{2} f_{\vec{p}}\left(x^{\prime}\right)$ and $Q_{i}^{\prime} f_{\vec{p}}\left(x^{\prime}\right)=p_{i} f_{\vec{p}}\left(x^{\prime}\right)$. We arrive thus to our principal conclusion:

Given the set of P-mode functions $f_{\vec{p}}$ in the chart $\{t, \vec{x}\}$, the functions $f_{\vec{p}} \circ \pi_{[0]}$ form the set of $Q$-mode functions of the same chart, depending on the parameters $\vec{q} \in R^{3}$

Obviously, these new mode functions are solutions of the Klein-Gordon equation and satisfy $Q_{i} f_{\vec{p}}\left[\pi_{[0]}(x)\right]=q_{i} f_{\vec{p}}\left[\pi_{[0]}(x)\right]$ where the operators $Q_{i}$ are given by equation (6).

Hence our problem is completely solved but this gives rise to other questions to be addressed in further investigations. It remains to study what happens with the similar modes of the fields with spin and how the discrete isometries act in this case. Moreover, the physical meaning of the $Q$-modes must be elucidated by working out significant examples.

\section{Acknowledgements}

This work is partially supported by the ICTP-SEENET-MTP grant PRJ-09 in frame of the SEENET-MTP Network G. S. Dj..

\section{References}

[1] S. Weinberg, Gravitation and Cosmology: Principles and Applications of the General Theory of Relativity (Wiley, New York, 1972).

[2] I. I. Cotăescu, Gen. Relativity Gravitation 43, 1639 (2011).

[3] N. D. Birrel and P. C. W. Davies, Quantum Fields in Curved Space (Cambridge University Press, Cambridge 1982).

[4] I. I. Cotăescu, J. Phys. A: Math. Gen. 33, 9177 (2000).

[5] B. Carter and R. G. McLenaghan, Phys. Rev. D 19, 1093 (1979).

[6] N. A. Chernikov and E. A. Tagirov, Ann. Inst H. Poincaré IX 1147 (1968); P. Candelas and D. J. Raine, Phys. Rev. D 12965 (1975); J. S. Dowker and J. S. Critchely, Phys. Rev. D 13, 224 (1976); T. S. Bunch and P. C. W. Davies, Proc. R. Soc. Lond. A 360, 117 (1978)

[7] I. I. Cotăescu, C. CruceaAn and A. Pop, Int. J. Mod. Phys. A 23, 2463 (2008). 